\title{
REMARKS ABOUT $\gamma$-SETS AND BOREL-DENSE SETS
}

\author{
IRENEUSZ RECLAW
}

(Communicated by Andreas R. Blass)

\begin{abstract}
We show, assuming Martin's Axiom, that every set of cardinality the continuum containing a Borel-dense set of cardinality less than the continuum is a $\gamma$-set but is not a hereditarily $\gamma$-set. This answers a question of D. H. Fremlin and J. Jasinski.
\end{abstract}

A family $J \subset P(X)$ is an $\omega$-cover of $X$ if for every finite set $F \subset X$ there exists $B \in J$ such that $F \subset B$. A topological space $X$ is a $\gamma$-set if for every open $\omega$-cover $J$ of $X$ there exists a family $\left\{D_{n}: n \in \omega\right\} \subset J$ such that $X \subset \bigcup_{m} \bigcap_{n>m} D_{n}$.

This definition was introduced by Gerlits and Nagy [3]. They showed that a space $X$ is a $\gamma$-set iff $C(X)$ is a Frechet space. Galvin and Miller [2] showed that under MA there is a $\gamma$-set of the reals of size continuum. Since every $\gamma$-set of reals is strong measure zero (see [3]), by Laver's result (see [4]) it is consistent that there is no uncountable $\gamma$-set of reals. Under $\diamond \omega_{1}$ Todorcevic, (see [2]) showed that there is a hereditarily $\gamma$-set of reals of size c. It is still an open problem whether MA or $\mathrm{CH}$ implies the existence of a hereditarily $\gamma$-set of reals of size $c$.

A subset $A$ of separable metric space $X$ is Borel-dense in $X$ if $A$ meets every Borel subset of cardinality continuum of $X$.

D. H. Fremlin and J. Jasinski [1] showed that if Martin's Axiom holds and there exists $\kappa<\mathbf{c}$ such that $P(\kappa)$ contains a proper, uniform, $\omega_{1}$-saturated, $\kappa$-additive ideal, then there exists a set $X$ of reals of cardinality the continuum containing a subset $D$ of cardinality less than the continuum Borel-dense in $X$. They also showed that $X$ can be a $\gamma$-set. They asked a question: Can $X$ be a hereditarily $\gamma$-set?

Theorem. Assume Martin's Axiom. If $X \subset \mathbf{R}$ of cardinality continuum contains a subset $D$ of cardinality less than continuum Borel-dense in $X$, then $X$ is a $\gamma$-set but is not a hereditarily $\gamma$-set.

Received by the editors January 12, 1994 and, in revised form, April 26, 1994.

1991 Mathematics Subject Classification. Primary 04A15; Secondary 03E50.

This research is partially supported by the Emmy Noether Institute in Mathematics of Bar Ilan University, Israel and by the Minerva Foundation, Germany.

(C)1995 American Mathematical Society 
Lemma 1. Assume Martin's Axiom. Let $X \subset \mathbf{R}$. If there exists $D \subset X$ such that $|D|<\mathbf{c}$ and for every Borel subset $B$ containing $D,|X \backslash B|<\mathbf{c}$, then $X$ is a $\gamma$-set.

Proof. Let $J$ be an open $\omega$-cover of $X$. We will show that there exists a family $\left\{B_{n}^{k}: n, k \in \omega\right\} \subset J$ such that for every $Y \in[X]^{<\omega}$ there exists $k \in \omega$ such that for infinitely many $n, Y \subset B_{n}^{k}$ and for every $k \in \omega, D \subset \bigcup_{m} \bigcap_{n>m} B_{n}^{k}$. We know that every set of reals of cardinality less than continuum is a $\gamma$-set (see [2]). There exists a family $\left\{B_{n}^{0}: n \in \omega\right\} \subset J$ such that $D \subset \bigcup_{m} \bigcap_{n>m} B_{n}^{0}$. Let $X_{1}=X \backslash \bigcup_{m} \bigcap_{n>m} B_{n}^{0}$. Then $\left|X_{1}\right|<\mathrm{c}$. Let $X_{1}, X_{2}, \ldots, X_{k} \subset X$ be such that $\left|X_{i}\right|<\mathbf{c}$ for $1 \leq i \leq k$. Then $\bigcup_{i=1}^{k} X_{i} \cup D$ is a $\gamma$-set. Thus there exists a family $\left\{B_{n}^{k}: n \in \omega\right\} \subset J$ such that $\bigcup_{i=1}^{k} X_{i} \cup D \subset \bigcup_{m} \bigcap_{n>m} B_{n}^{k}$, and we let $X_{k+1}=X \backslash \bigcup_{m} \bigcap_{n>m} B_{n}^{k}$. It is not hard to see that the family $\left\{B_{n}^{k}: n, k \in \omega\right\}$ has the required properties.

For $d \in D$ there exists a function $f_{d}: \omega \rightarrow \omega$ such that if $f_{d}(k)=m$, then $d \in \bigcap_{n \geq m} B_{n}^{k}$. Since $|D|<\mathbf{c}$, there exists a function $g: \omega \rightarrow \omega$ such that $f_{d}<^{*} g$. Let $J_{0}=\left\{B_{n}^{k}: n>g(k)\right.$ and $\left.k, n \in \omega\right\}=\left\{C_{n}: n \in \omega\right\}$. Observe that $D \subset \bigcup_{m} \bigcap_{n>m} C_{n}$. Because for every $d \in D$ there exists $l \in \omega$ such that if $k>l$ and $n>g(k)$, then $d \in B_{n}^{k}$, if $k \leq l$, then there exist only finitely many $n$ such that $d \notin B_{n}^{k}$. Thus $d$ must be in all but finitely many $C_{n}$. We know also that every finite subset of $X$ is contained in infinitely many $C_{n}$. Let $Z=X \backslash \bigcup_{m} \bigcap_{n>m} C_{n}$. Evidently $|Z|<\mathbf{c}$. If $|Z|<\omega$, then there exists an increasing sequence $n_{k}$ such that $Z \subset C_{n_{k}}$ for every $k$. Thus $X \subset \bigcup_{m} \bigcap_{k>m} C_{n_{k}}$. If $|Z| \geq \omega$, then $\left\{C_{n} \backslash\left\{y_{n}\right\}: n \in \omega\right\}$ is an $\omega$-cover of $X$ for a sequence $\left\{y_{n}: n \in \omega\right\} \subset Z$ of distinct elements. Thus there exists an increasing sequence $n_{k}$ such that $Z \subset \bigcup_{m} \bigcap_{k>m} C_{n_{k}}$, so $X \subset \bigcup_{m} \bigcap_{k>m} C_{n_{k}}$.

Lemma 2. Assume Martin's Axiom. Let $X \subset(0,1)$ and $D, D_{1} \subset(1,2)$ be such that $|D|=\left|D_{1}\right|<\mathbf{c}$. Then $X \cup D$ is a $\gamma$-set iff $X \cup D_{1}$ is a $\gamma$-set.

Proof. Assume that $X \cup D$ is a $\gamma$-set. Let $g: D \rightarrow D_{1}$ be a bijection and $f: X \cup D \rightarrow X \cup D_{1}, f(x)=x$ if $x \in X$ and $g(x)$ if $x \in D$. Let $J=$ $\left\{O^{n}: n \in \omega\right\}$ be an open $\omega$-cover of $X \cup D_{1}$. Let $O^{n}=O_{1}^{n} \cup O_{2}^{n}$ where $O_{1}^{n} \subset X, O_{2}^{n} \subset D_{1}$. Then $\left\{f^{-1}\left(O^{n}\right): n \in \omega\right\}$ is an $\omega$-cover of $X \cup D$, and we let $f^{-1}\left(O^{n}\right)=f^{-1}\left(O_{1}^{n}\right) \cup f^{-1}\left(O_{2}^{n}\right)=G_{1}^{n} \cup G_{2}^{n}$ where $G_{1}^{n} \subset X$ is open and $G_{2}^{n} \subset D G_{\delta}$-set. There exists a family of open subsets of $D,\left\{H_{k}^{n}: n, k \in \omega\right\}$, such that for every $n, k H_{k+1}^{n} \subset H_{k}^{n}$ and $G_{2}^{n}=\bigcap_{k} H_{k}^{n}$.

For $d \in D$ let $h_{d}: \omega \rightarrow \omega$ be such that for every $n$, if $d \notin G_{2}^{n}$, then $h_{d}(n)=m$ such that $d \notin H_{m}^{n}$ and if $d \in G_{2}^{n}$, then $h_{d}(n)=0$. Since $|D|<\mathbf{c}$, there exists a function $h: \omega \rightarrow \omega$ such that for every $d \in D, h_{d}<^{*} h$. Observe that $\left\{G_{1}^{n} \cup H_{h(n)}^{n}: n \in \omega\right\}$ is an open $\omega$-cover of $X \cup D$ so there exists an increasing sequence $n_{k}$ such that $X \cup D=\bigcup_{m} \bigcap_{k>m} G_{1}^{n_{k}} \cup H_{h\left(n_{k}\right)}^{n_{k}}$. We will show that $X \cup D=\bigcup_{m} \bigcap_{k>m} G_{1}^{n_{k}} \cup G_{2}^{n_{k}}$. Evidently $X \subset \cup_{m} \bigcap_{k>m} G_{1}^{n_{k}} \cup$ $G_{2}^{n_{k}}$. Suppose that there exists $d \in D$ such that $\forall_{m} \exists_{k \geq m} d \notin G_{2}^{n_{k}}$. But we know that $\exists_{m} \forall_{k \geq m} d \notin G_{2}^{n_{k}} \Rightarrow d \notin H_{h\left(n_{k}\right)}^{n_{k}}$, so $\forall_{m} \exists_{k \geq m} d \notin H_{h\left(n_{k}\right)}^{n_{k}}$, thus $d \notin$ $\bigcup_{m} \bigcap_{k>m} H_{h\left(n_{k}\right)}^{n_{k}}$.

It follows that

$$
X \cup D_{1}=\bigcup_{m} \bigcap_{k>m} O^{n_{k}}
$$


Lemma 3. Assume Martin's Axiom. If every subset of $X \subset \mathbf{R}$ is a $\gamma$-set, then for every $D$ of cardinality less than continuum $D$ is $F_{\sigma}$ and $G_{\delta}$ in $X \cup D$.

Proof. If $|X|<\mathbf{c}$, then the proof is well known. Assume that $|X|=\mathbf{c}$. Suppose that there exists $D$ such that $|D|<\mathrm{c}$ and $D$ is not $G_{\delta}$ or not $F_{\sigma}$ in $X \cup D$. Then there exists an interval $(a, b)$ such that $D \cap(a, b)$ is not $G_{\delta}$ or not $F_{\sigma}$ in $(X \cup D) \cap(a, b)$ and $|X \backslash(a, b)|=\mathbf{c}$. There exists $D_{1} \subset X \backslash(a, b)$ such that $\left|D_{1}\right|=|D \cap(a, b)|$. From Theorem 3 of Galvin and Miller [2] $[(X \cap(a, b)) \backslash D] \cup[(D \cap(a, b))+(b-a)]$ is not a $\gamma$-set. Thus from Lemma 2 $((X \cap(a, b)) \backslash D) \cup D_{1}$ is also not a $\gamma$-set but $\left[((X \cap(a, b)) \backslash D) \cup D_{1}\right] \subset X$.

$D$ in the Theorem is not Borel in $X$, so from Lemmas 1 and 3 we get the theorem.

\section{REFERENCES}

1. D. H. Fremlin and J. Jasinski, $G_{\delta}$-covers and large thin set of real numbers, Proc. London Math. Soc. (3) 53 (1986), 518-538.

2. F. Galvin and A. W. Miller, $\gamma$-sets and other singular sets of real line, Topology Appl. 17 (1984), 145-155.

3. J. Gerlits and Zs. Nagy, Some properties of $C(X)$. I, Topology Appl. 14 (1982), 151-161.

4. R. Laver, On consistency of Borel's conjecture, Acta. Math. 137 (1976), 151-169.

Institute of Mathematics, University of Gdansk, Wita Stwosza 57, 80-952 Gdansk, POLAND

E-mail address: matir@halina.univ.gda.pl 